\title{
Controlled trial of antituberculous chemotherapy in Crohn's disease: a five year follow up study
}

G A O Thomas, G L Swift, J T Green, R G Newcombe, C Braniff-Mathews, J Rhodes, $S$ Wilkinson, G Strohmeyer, G Kreuzpainter disease, height, weight, CDAI, and blood tests. Thirty seven patients in the active group and 30 in the placebo group had undergone previous surgical procedures. There was no difference in concomitant treatment between the two groups. Thirty patients in the active group and 46 in the placebo group were taking corticosteroids at entry to the trial. Forty eight of 63 patients in the active and 49 of 63 in the placebo group completed at least 12 months' therapy. Reasons for early withdrawal included pregnancy, adverse reaction, and failure to comply. There was no significant difference in the mean number of months completed between the two groups. Nineteen adverse reactions were recorded for 17 patients in the active group, compared with three in the placebo group; all nine patients withdrawn early because of adverse reactions were in the active group. The effect of treatment on the course of the disease during the two year study was assessed in a number of ways, which included comparison between groups of the number of episodes of surgery required, radiological change, and the total steroid dosage during the study; the number of days on which the prednisolone dose was $10 \mathrm{mg}$ or greater was also compared, as were changes in disease activity based on the Harvey-Bradshaw Index ${ }^{7}$ and blood tests.

Fifteen patients on active treatment compared with 14 on placebo had surgery during the trial, with no difference in the type of surgery required between the groups. Radiological assessments based on 98 patients at the end of the trial showed no significant differences between groups in changes of extent of disease. More patients developed strictures on placebo than active treatment but without a statistically significant difference. No differences were found between groups for the total prednisolone dose or the number of days on which the prednisolone dose was $10 \mathrm{mg}$ or more. Serial measurements of body weight and Crohn's disease activity index (CDAI), together with blood values for albumin, haemoglobin, white cell count, and platelets, showed no consistent differences between groups. There were occasional significant differences for some of these values between groups, which were not sustained. In conclusion, comparison of the various outcome measurements between groups gave little evidence of tangible benefit from the trial treatment at the end of the two years.

These patients have subsequently been followed for a total of five years from entry to 
the trial, and the clinical outcome in the two groups compared.

\section{Methods}

To examine the longer term effect of antituberculous treatment given during the first two years, patients were followed for a total of five years from their date of entry to the study. They were seen routinely in the outpatient department at times determined by clinical need and also at yearly intervals. Patients continued on standard therapy throughout this period, the nature of which depended on the disease site, type, and activity. Treatment included various combinations of 5-acetylsalicylic acid preparations, steroids, immunosuppressants, and antibiotics. These were used in a routine way to treat both active episodes and maintain remission. At each annual review, events of the preceding 12 months were documented in a manner similar to that used in the initial 24 month study period. Specifically, the number of acute relapses of Crohn's disease, defined as commencement or increase in the dose of prednisolone to control symptoms, was recorded; a note was also made of whether the patient had undergone surgery for the disease, and if so the type was documented. The number of hospital admissions for Crohn's disease over the preceding 12 months, the disease activity index, full blood count, albumin, and current medication were also recorded.

The principal outcome measures of the follow up assessments were the number of clinical relapses, the number of patients who had surgery for Crohn's disease over the preceding year, and the total disease activity score at each visit.

The analyses performed were simple comparisons of the active and placebo groups at 36, 48 , and 60 months, and did not take into account any data collected at baseline or during the course of the first two years of the

Table 1 Details of patients given antituberculous treatment for up to 2 years and followed for 5 years from entry to the study

\begin{tabular}{|c|c|c|c|}
\hline & \multicolumn{3}{|c|}{ Months from entry to study } \\
\hline & 36 & 48 & 60 \\
\hline No of patients (active/placebo) & $52 / 57$ & $49 / 51$ & $38 / 50$ \\
\hline \multicolumn{4}{|l|}{ Disease extent (active/placebo) } \\
\hline Small bowel & $16 / 19$ & $15 / 18$ & $10 / 17$ \\
\hline Large bowel & $14 / 14$ & $14 / 14$ & $11 / 11$ \\
\hline Mixed & $22 / 24$ & $20 / 19$ & $17 / 22$ \\
\hline Number of acute relapses (active/placebo) & $29 / 35$ & $19 / 22$ & $10 / 26$ \\
\hline Surgery (active/placebo) ${ }^{\star}$ & $10 / 11$ & $5 / 7$ & $3 / 9$ \\
\hline Perianal & $3 / 2$ & $2 / 3$ & $0 / 0$ \\
\hline Laparotomy & $1 / 1$ & $0 / 0$ & $1 / 1$ \\
\hline Right hemicolectomy & $0 / 2$ & $0 / 1$ & $1 / 0$ \\
\hline Ileocaecectomy & $5 / 2$ & $1 / 0$ & $1 / 5$ \\
\hline Partial colectomy & $1 / 3$ & $1 / 0$ & $0 / 0$ \\
\hline Total colectomy & $0 / 1$ & $1 / 1$ & $0 / 1$ \\
\hline Stricturoplasty & $0 / 2$ & $1 / 2$ & $0 / 0$ \\
\hline Small bowel & $0 / 0$ & $0 / 0$ & $1 / 2$ \\
\hline Admission (active/placebo) & $12 / 15$ & $10 / 9$ & $6 / 6$ \\
\hline Total disease activity (active/placebo) $\dagger$ & $5 / 4$ & $3 / 5$ & $5 / 4$ \\
\hline \multicolumn{4}{|l|}{ Blood results (active/placebo) } \\
\hline Haemoglobin $(\mathrm{g} / \mathrm{dl})$ & $13 / 12$ & $13 / 13$ & $13 / 13$ \\
\hline White cell count $\left(\times 10^{9} / 1\right)$ & $8 / 8$ & $8 / 8$ & $9 / 9$ \\
\hline Platelet count $\left(\times 10^{9} / 1\right)$ & $291 / 302$ & $291 / 329$ & $290 / 330$ \\
\hline Albumin $(\mathrm{g} / \mathrm{l})$ & $43 / 44$ & $44 / 44$ & $41 / 42$ \\
\hline
\end{tabular}

${ }^{\star}$ A number of patients had two surgical procedures, thus in some cases the numbers are greater than the total number of patients who underwent surgery.

$\dagger$ Disease activity score is based on a modification of the Harvey-Bradshaw Index ${ }^{7}$ with a maximum total score of 17 plus the number of motions $/ 24 \mathrm{~h}$. study. The most sensitive test of long term efficacy would be the cumulative number of relapses; however, due to missing data in the long term follow up this was not possible. Unpaired $t$ tests, Mann-Whitney, or $\chi^{2}$ tests were used to compare data between groups where appropriate.

\section{Results}

One hundred and thirty patients entered the initial study. Of these, $111(81 \%)$ were followed regularly for five years from the time of entry to the study; follow up data for the remaining 19 patients were inadequate or incomplete and therefore were not included. Of the 111 patients, the number with records at 36, 48, and 60 months was 109,100 , and 88 respectively; other records were unavailable or insufficient. There were no deaths in the three years following completion of treatment; three patients died during the first 24 months - two in the active and one in the placebo group as reported previously.

NUMBER OF ACUTE RELAPSES OF CROHN'S

DISEASE

At 60 months there was a nominal, statistically significant advantage for the active group $(p=0.04) ; 10 / 38(26 \%)$ of the active group, and $26 / 50(52 \%)$ of the control group, had one or more relapses in the preceding year. At 36 months, $29 / 52(56 \%)$ of the active versus $35 / 57$ $(61 \%)$ of the placebo group, and at 48 months, $19 / 49$ (39\%) versus $22 / 51$ (43\%) respectively had suffered at least one relapse. Although these figures tend to favour active treatment, the difference is minimal and only statistically significant at one time point in 60 months.

NUMBER OF SURGICAL EPISODES

There was no significant difference between treatment groups in the number who had surgery for Crohn's disease over the previous 12 months at 36,48 , and 60 months: active $10 / 52$ (19\%) versus control 11/57 (19\%); 5/49 (10\%) versus $7 / 51$ (14\%); and $3 / 38(8 \%)$ versus $9 / 50$ $(18 \%)$ respectively. The nature of surgery was also similar for both groups at all time points (see table 1); procedures included perianal surgery, resections of ileum and colon, and stricturoplasties. In some cases, patients may have had more than one operative procedure, hence the total number of procedures may be greater than the number of patients undergoing surgery.

NUMBER OF HOSPITAL ADMISSIONS

There was no significant difference between treatment groups in the number who were admitted to hospital with Crohn's disease at 36, 48, and 60 months: active 12/52 (23\%) versus control 15/57 (26\%); 10/49 (20\%) versus $9 / 51(17 \%)$; and $6 / 38(16 \%)$ versus $6 / 50$ $(12 \%)$ respectively.

DISEASE ACTIVITY, BLOOD TESTS, AND MEDICATION

There was no significant difference between treatment groups for disease activity at 36,48 , and 60 months. There were small but inconsistent differences between groups for various 
blood tests such as platelets and albumin. The total dose of steroid used by patients in both groups was similar at all stages. Likewise, there were no consistent or sustained differences in other medications used to treat Crohn's disease at any stage.

\section{Discussion}

We report the five year follow up of a two year study of triple antituberculous chemotherapy. Although the number of relapses in those given active treatment was less than in the placebo group during the follow up period, the results only just achieved statistical significance at 60 months. There were other occasional significant differences between the groups for some of the other values, but none were consistent or sustained, and overall there was no other evidence of tangible benefit or disadvantage from antituberculous chemotherapy in any of the assessments made including the number of surgical episodes, hospital admissions, disease activity, and medication required for Crohn's disease.

There are a number of obvious limitations with this study. We acknowledge that the most sensitive test of long term efficacy would be the cumulative relapse rate; however, due to missing data this cannot be calculated in any meaningful way-a problem observed with other long term follow up studies. We outlined in our original report the difficulties associated with clinical trials of this kind in patients with Crohn's disease. ${ }^{6}$ It is quite possible that the response to therapy depends not only on the site of the disease, but also the type-for instance, whether the patient has predominantly stricturing, fistulating, or inflammatory disease. In future studies, disease should be stratified not only according to site but also type; this was not done in our study. With the benefit of hindsight the choice of antituberculous chemotherapy may be questioned. It is now eight years since the commencement of this study. During this time it has been recognised that some species of mycobacteria are resistant to conventional antituberculous treatment regimens; these include $M$ paratuberculosis, the cause of Johne's disease in cattle and a possible cause of Crohn's disease. We chose rifampicin, ethambutol, and isoniazid after preliminary studies indicated that $M$ paratuberculosis was sensitive to this combination of antituberculous drugs. The in vitro tests were carried out in the Mycobacterium Reference Unit of the Public Health Laboratory Service at the Cardiff Public Health Laboratory using a conventional model resistance method. The correlation between in vitro sensitivity of tubercle bacilli to antituberculous drugs and the in vivo response to treatment with these drugs has been well established by many controlled clinical trials. This is not the case with disease due to atypical mycobacteria such as $M$ paratuberculosis. In addition, organisms of the Avium intracellulare complex which is closely related to $M$ paratuberculosis are highly resistant in vitro to rifampicin and ethambutol but paradoxically a significant number of patients respond clinically. Even in situations where a mycobacterium has been identified as the cause of a disease, in vitro sensitivity tests are not necessarily a reliable guide to clinical response. The choice of antibiotics for atypical mycobacterial infections remains difficult and controversial.

Although conventional treatment was used in addition to trial medication throughout the five year period, this should not have influenced the results. Both groups of patients continued on standard medication which was used in a routine manner to treat active symptoms, maintain clinical remission, and was tailored to disease activity and site. There was no difference between groups in the type of medication taken at different time points or in the steroid dose used.

The primary objective of this trial was to examine whether there was net benefit for patients given triple antituberculous chemotherapy for two years. The absence of any benefit at the end of the two year trial period, and furthermore, after three years of subsequent follow up, fails to support the hypothesis that mycobacteria play an important part in the pathogenesis of this disease. It is not possible to say from this study whether there may be benefit in certain subgroups, for example those with stricturing disease, but with the available patient data it would be wrong and unhelpful to perform subgroup analyses in retrospect.

There have been anecdotal reports in the literature of clinical improvement in patients with Crohn's disease given antituberculous treatment, ${ }^{458}$ but randomised and open studies have yielded less convincing results. A total of 368 patients have taken part in nine published trials of antituberculous treatment in Crohn's disease. ${ }^{6}{ }^{10}$ Seven were randomised and placebo controlled, ${ }^{6911}$ and two were open. ${ }^{1012}$ During these studies, treatment was given for three to 24 months, and various antituberculous regimens were used, from single to quadruple regimens. Five of the studies, including our own, showed no significant clinical benefit from active treatment. ${ }^{6-11}{ }^{9-15}$ Two studies showed possible benefit; of these one was a very small study in which clarithromycin alone was used $^{12}$ while in the other, quadruple therapy was given. Although clinical relapses were fewer on active drugs, there were no differences in endoscopic or surgical recurrence. ${ }^{16}$ It is possible that the immediate improvement reported in some studies may be due to an effect of antibiotics on intestinal flora, rather than a direct effect on mycobacteria; in this case, one would not expect lasting benefit. Likewise, failure to improve with treatment initially does not exclude the possible role of mycobacteria initiating the disease. However, we have previously suggested that extension or recurrence of Crohn's disease after a period of treatment may be a better indicator of a therapeutic effect on the underlying process-hence the need for an extended five year assessment. The complexity of Crohn's disease as well as differences in the regimens used, make evaluation and comparison of trials difficult. However, a large number of patients have now been treated with antituberculous drugs for a significant period of time, and at 
present there are few data to suggest significant clinical benefit or support for the long term use of potentially toxic drugs.

1 Stanford JL, White SA, Burnham WR, et al. Mycobacteria and inflammatory bowel disease. Lancet 1979;i:444.

2 Graham DY, Markesich DC, Yoshimura HH. Mycobacteria and inflammatory bowel disease; results of culture. Gastroenterology 1987;92:436-42.

3 Morgan KL. Johne's and Crohn's; chronic inflammatory bowel diseases of infectious aetiology? Lancet 1987; i:1017-

4 Shultz MG, Reider HL, Hersh T, et al. Remission of Crohn's disease with anti-mycobacterial chemotherapy. Lancet disease with anti-mycobacterial chemotherapy. Lancet 1987;ii:1391-2.

Picciotto A, Gesu GP, Schito GC, et al. Antimycobacterial chemotherapy in 2 cases of inflammatory bowel disease. Lancet 1988,1i:536-7.

6 Swift GL, Srivastava ED, Stone R, et al. Controlled trial of anti-tuberculous chemotherapy for two years in Crohn's disease. Gut 1994;35:363-8.

7 Best ER, Bektel JM, Singleton JW, et al. Development of a Crohn's Disease Activity Index: National Cooperative Crohn's Disease Study. Gastroenterology 1976;70:439-44.

8 Warren JB, Rees HC, Cox TM. Remission of Crohn's disease with tuberculosis chemotherapy. $N$ Engl f $\mathrm{Med}$ 1986;314:182.
9 Elliot PR, Burnham WR, Berghouse LM, et al. Sulphadoxine-pyrimethamine therapy in Crohn's disease. Digestion 1982;23:132-4

10 Rutgeerts P, Geboes K, Vantrappen G, et al. Rifabutin and ethambutol do not help recurrent Crohn's disease in the neoterminal ileum. F Clin Gastroenterol 1992;15:24-8.

11 Graham DY, Al-Assi MT, Robinson M. Prolonged remission in Crohn's disease following therapy for Mycobacterium paratuberculosis [abstract]. Gastroenterology 1995; 108:A826.

12 Hampson SJ, Parker MC, Saverymuttu SH, et al. Quadruple anti-mycobacterial chemotherapy in Crohn's disease; results at 9 months of a pilot study in 20 patients. Aliment Pharmacol Ther 1989;3:343-52.

13 Shaffer JL, Hughes S, Linaker BD, et al. Controlled trial of rifampicin and ethambutol in Crohn's disease. Gut 1984;25:203-5.

14 Basilisco G, Ranzi T, Campanini C, et al. Controlled trial of rifabutin in Crohn's disease. Curr Ther Res 1989;46:24250.

15 Afdhal NH, Long A, Lennon J, et al. Controlled trial of antimycobacterial therapy in Crohn's disease. Clofazimine versus placebo. Dig Dis Sci 1991;36:449-53.

16 Prantera C, Kohn A, Mangiarotti R, et al. Antimycobacterial therapy in Crohn's disease; results of a controlled, double-blind trial with a multiple antibiotic regimen. Am f Gastroenterol 1994;89:513-18. 